\title{
Sauter-Schwinger like tunneling in tilted Bose-Hubbard lattices in the Mott phase
}

\author{
Friedemann Queisser ${ }^{1}$, Patrick Navez ${ }^{2}$, and Ralf Schützhold ${ }^{1, *}$ \\ ${ }^{1}$ Fakultät für Physik, Universität Duisburg-Essen, Lotharstrasse 1, 47057 Duisburg, Germany \\ ${ }^{2}$ Institut für Theoretische Physik, Technische Universität Dresden, 01062 Dresden, Germany
}

(Dated: September 6, 2021)

\begin{abstract}
We study the Mott phase of the Bose-Hubbard model on a tilted lattice. On the (Gutzwiller) mean-field level, the tilt has no effect - but quantum fluctuations entail particle-hole pair creation via tunneling. For small potential gradients (long-wavelength limit), we derive a quantitative analogy to the Sauter-Schwinger effect, i.e., electron-positron pair creation out of the vacuum by an electric field. For large tilts, we obtain resonant tunneling related to Bloch oscillations.
\end{abstract}

PACS numbers: 67.85.-d, 12.20.-m, 05.30.Rt.

Introduction. There are many striking analogies between apparently very different areas in physics, which help us to unify our understanding of nature - as R. Feynman said: The same equations have the same solutions. In this Letter, we establish such a quantitative analogy between high-field science and ultra-cold atoms in optical lattices. The Sauter-Schwinger effect [1-3] predicts that an extremely strong electric field may create electronpositron pairs out of the QED vacuum. As an intuitive picture, one can envisage this effect as tunneling of an electron from the Dirac sea into the positive continuum, leaving behind a hole (i.e., positron), see Fig. 11. Unfortunately, this prediction has not been experimentally verified yet since it is hard to generate sufficiently strong electric fields in large enough space-time regions. As we shall demonstrate below, the same equations govern particlehole pair creation in a Mott insulator via tunneling due to a small potential gradient. The Mott insulator state we are considering can be generated by bosonic atoms in an optical lattice [4, 5] for example, which repel each other and thereby become effectively pinned to the lattice sites (zero mobility). The benefits of this quantitative analogy are two-fold. On the one hand, we can apply our knowledge of the Sauter-Schwinger effect [1, 2] to atoms in optical lattices and understand them better in this way. On the other hand, atoms in optical lattices may provide (via this analogy) an experimental approach to the so far unobserved Sauter-Schwinger effect. a)

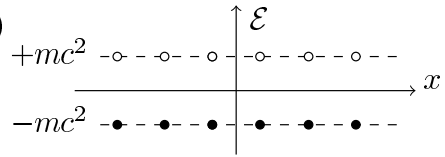

c)

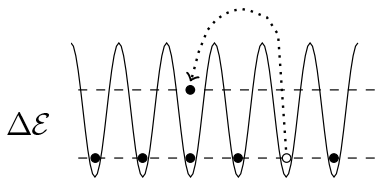

b)

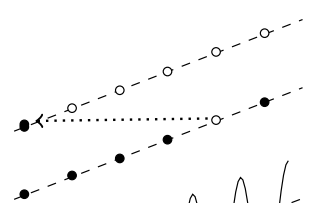

d)

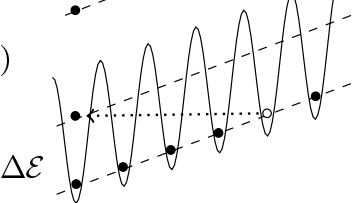

FIG. 1: Sketch of the analogy: a) Dirac sea for $E=0$, b) Sauter-Schwinger tunneling for $E \neq 0$, c) Mott state with energy gap $\Delta \mathcal{E}$, d) tunneling in tilted lattice.
Sauter-Schwinger effect. Let us start with the Dirac equation describing relativistic electrons $(\hbar=c=1)$

$$
\gamma^{\mu}\left(i \partial_{\mu}-q A_{\mu}\right) \Psi-m \Psi=0,
$$

with charge $q$ and mass $m$, propagating in an electromagnetic field $A_{\mu}$. An electric field $\boldsymbol{E}$ can be encoded in the scalar potential $\boldsymbol{E}=\boldsymbol{\nabla} \Phi$ or be generated by the vector potential $\boldsymbol{E}=\partial_{t} \boldsymbol{A}$. Using the standard representation of the Dirac matrices $\gamma^{\mu}$ via the Pauli matrices $\boldsymbol{\sigma}$ and splitting the bi-spinor $\Psi$ into upper and lower components $\Psi=\left(\psi_{+}, \psi_{-}\right)$, the "square" of Eq. (1) yields

$$
\left[\left(i \partial_{t}-q \Phi\right)^{2}-(i \boldsymbol{\nabla}+q \boldsymbol{A})^{2}-m^{2}\right] \psi_{ \pm}=i q \boldsymbol{E} \cdot \boldsymbol{\sigma} \psi_{\mp} .
$$

For sub-critical electric fields $m^{2} \gg q E$, we may neglect the spin-dependent term on r.h.s. and obtain the KleinFock-Gordon equation for both spinors $\psi_{ \pm}$separately. If the electric field is purely time-dependent $\boldsymbol{E}=E(t) \boldsymbol{e}_{x}$, the temporal gauge $\Phi=0$ and $\boldsymbol{E}=\partial_{t} \boldsymbol{A}$ is most convenient: After a spatial Fourier transform, we find that each mode $\boldsymbol{k}$ corresponds to a harmonic oscillator

$$
\left[\frac{d^{2}}{d t^{2}}+\left[k_{x}+q A(t)\right]^{2}+\boldsymbol{k}_{\perp}^{2}+m^{2}\right] \psi_{\boldsymbol{k}}=0,
$$

with a time-dependent potential. Replacing $t$ by $x$, the above equation is equivalent to a one-dimensional Schrödinger scattering problem. A solution which initially behaves as $\psi_{\boldsymbol{k}}^{\text {in }}(t) \propto \exp \left\{-i \omega_{\boldsymbol{k}} t\right\}$ will finally evolve into a mixture of positive and negative frequencies $\psi_{\boldsymbol{k}}^{\text {out }}(t) \propto \alpha_{\boldsymbol{k}} \exp \left\{-i \omega_{\boldsymbol{k}} t\right\}+\beta_{\boldsymbol{k}} \exp \left\{+i \omega_{\boldsymbol{k}} t\right\}$. The Bogoliubov coefficient $\beta_{\boldsymbol{k}}$ is related to the reflection amplitude in one-dimensional scattering theory and yields the probability for electron-positron pair creation. For slowly varying fields $E(t)$ with $m^{2} \gg q E$, it can be derived via the WKB approximation. Since Eq. (3) corresponds to scattering above the barrier, the turning points are not real but complex. Hence the reflection (i.e., pair creation) probability is exponentially suppressed [1] demonstrating that it is a non-perturbative effect

$$
P_{\boldsymbol{k},-\boldsymbol{k}}^{e^{+} e^{-}}=\left|\beta_{\boldsymbol{k}}^{2}\right| \propto \exp \left\{-\pi \frac{m^{2}+\boldsymbol{k}_{\perp}^{2}}{q E}\right\},
$$

where $E$ denotes the maximum electric field. 
Bose-Hubbard model. We consider the Hamiltonian

$$
\hat{H}=-\frac{J}{Z} \sum_{\mu, \nu} T_{\mu \nu} \hat{a}_{\mu}^{\dagger} \hat{a}_{\nu}+\frac{U}{2} \sum_{\mu}\left(\hat{a}_{\mu}^{\dagger}\right)^{2} \hat{a}_{\mu}^{2}+\sum_{\mu} V_{\mu} \hat{n}_{\mu},
$$

with the creation and annihilation operators $\hat{a}_{\mu}^{\dagger}$ and $\hat{a}_{\nu}$ at the lattice sites $\mu$ and $\nu$, respectively. The tunneling matrix $T_{\mu \nu} \in\{0,1\}$ encodes the lattice structure and $J$ denotes the hopping rate. The number of tunneling neighbors at any given site $\mu$ yields the coordination number $Z=\sum_{\nu} T_{\mu \nu} \in \mathbb{N}$. Finally, $U$ is the on-site interaction and $V_{\mu}$ denotes the on-site potential with $\hat{n}_{\mu}=\hat{a}_{\mu}^{\dagger} \hat{a}_{\mu}$. This potential $V_{\mu}$ at lattice position $\boldsymbol{r}_{\mu}$ will be analogous to the electric field $\boldsymbol{E}$ via $V_{\mu}(t) \leftrightarrow q \boldsymbol{r}_{\mu} \cdot \boldsymbol{E}(t)$.

We assume an average filling of one boson per site $\left\langle\hat{n}_{\mu}\right\rangle=1$. In order to have well-defined initial and final states, we envisage the following sequence: Initially, we have $J=V_{\mu}=0$ where the ground state simply reads $\left|\Psi_{\text {in }}\right\rangle=\prod_{\mu}|1\rangle_{\mu}$ (Mott insulator). Then we switch on the hopping rate $J$ adiabatically (i.e., slow compared to the energy gap of the Mott insulator) such that we stay in the ground state. Next we introduce the lattice potential $V_{\mu}(t)$ which will be analogous to the electric field and enables particle-hole creation via tunneling. Again, we should do this slowly in order to avoid dynamical excitations [2]. Finally, we reverse this process and slowly switch off first $V_{\mu}(t)$ and then $J$. Thus the final ground state again reads $\prod_{\mu}|1\rangle_{\mu}$. If the final quantum state does not have exactly one particle per site $\left|\Psi_{\text {out }}\right\rangle \neq \prod_{\mu}|1\rangle_{\mu}$ we have a signature of particle-hole pair creation.

In order to calculate this effect, we proceed along the lines of [6] and consider the time evolution of the density operator $\hat{\rho}$ of the whole lattice and introduce Liouville super-operators $\widehat{\mathcal{L}}_{\mu}$ and $\widehat{\mathcal{L}}_{\mu \nu}$ via

$$
i \partial_{t} \hat{\rho}=[\hat{H}, \hat{\rho}]=\frac{1}{Z} \sum_{\mu, \nu} \widehat{\mathcal{L}}_{\mu \nu} \hat{\rho}+\sum_{\mu} \widehat{\mathcal{L}}_{\mu} \hat{\rho} .
$$

As the next step, we derive the reduced density matrices for one lattice site $\hat{\rho}_{\mu}=\operatorname{Tr}_{\mu}\{\hat{\rho}\}$ via averaging (tracing) over all other sites $\nu \neq \mu$ and similarly for two and more sites $\hat{\rho}_{\mu \nu}=\operatorname{Tr}_{\mu \nu}\{\hat{\rho}\}$ etc. Then we separate the correlated parts via $\hat{\rho}_{\mu \nu}=\hat{\rho}_{\mu \nu}^{\text {corr }}+\hat{\rho}_{\mu} \hat{\rho}_{\nu}$ for two sites, as well as $\hat{\rho}_{\mu \nu \lambda}=\hat{\rho}_{\mu \nu \lambda}^{\text {corr }}+\hat{\rho}_{\mu \nu}^{\text {corr }} \hat{\rho}_{\lambda}+\hat{\rho}_{\mu \lambda}^{\text {corr }} \hat{\rho}_{\nu}+\hat{\rho}_{\nu \lambda}^{\text {corr }} \hat{\rho}_{\mu}+\hat{\rho}_{\mu} \hat{\rho}_{\nu} \hat{\rho}_{\lambda}$ etc. From Eq. (6), we obtain the effective evolution equation for one lattice site $\mu$ via tracing over the rest $\nu \neq \mu$

$$
i \partial_{t} \hat{\rho}_{\mu}=\frac{1}{Z} \sum_{\kappa \neq \mu} \operatorname{Tr}_{\kappa}\left\{\widehat{\mathcal{L}}_{\mu \kappa}^{S}\left(\hat{\rho}_{\mu \kappa}^{\text {corr }}+\hat{\rho}_{\mu} \hat{\rho}_{\kappa}\right)\right\}+\widehat{\mathcal{L}}_{\mu} \hat{\rho}_{\mu},
$$

where $\widehat{\mathcal{L}}_{\mu \nu}^{S}=\widehat{\mathcal{L}}_{\mu \nu}+\widehat{\mathcal{L}}_{\nu \mu}$. In order to employ a controlled analytic approach, we consider the limit of large coordination numbers $Z \gg 1$. In this limit, the correlations obey the following hierarchy [6, 7]. While the one-site density matrix $\hat{\rho}_{\mu}=\mathcal{O}\left(Z^{0}\right)$ has entries of order one $\operatorname{Tr}_{\mu}\left\{\hat{\rho}_{\mu}\right\}=1$, the two-site correlations are suppressed via $\hat{\rho}_{\mu \nu}^{\text {corr }}=\mathcal{O}(1 / Z)$, the three-site correlations via $\hat{\rho}_{\mu \nu \lambda}^{\text {corr }}=\mathcal{O}\left(1 / Z^{2}\right)$ and so on. Thus, Eq. (7) reproduces the Gutzwiller ansatz to lowest order in $1 / Z$, which reads $\hat{\rho}_{\mu}=|1\rangle_{\mu}\langle 1|+\mathcal{O}(1 / Z)=\hat{\rho}_{\mu}^{0}+\mathcal{O}(1 / Z)$ in the Mott state. Inserting this result and neglecting terms of order $\mathcal{O}\left(1 / Z^{2}\right)$, we get for two sites [6]

$$
\begin{aligned}
i \partial_{t} \hat{\rho}_{\mu \nu}^{\text {corr }}= & \frac{1}{Z} \sum_{\kappa \neq \mu, \nu} \operatorname{Tr}_{\kappa}\left\{\widehat{\mathcal{L}}_{\mu \kappa}^{S} \hat{\rho}_{\nu \kappa}^{\text {corr }} \hat{\rho}_{\mu}^{0}+\widehat{\mathcal{L}}_{\nu \kappa}^{S} \hat{\rho}_{\mu \kappa}^{\text {corr }} \hat{\rho}_{\nu}^{0}\right\}+ \\
& +\left(\widehat{\mathcal{L}}_{\mu}+\widehat{\mathcal{L}}_{\nu}\right) \hat{\rho}_{\mu \nu}^{\mathrm{corr}}+\frac{1}{Z} \widehat{\mathcal{L}}_{\mu \nu}^{S} \hat{\rho}_{\mu}^{0} \hat{\rho}_{\nu}^{0} .
\end{aligned}
$$

Formally, this is an evolution equation for an infinite dimensional matrix $\hat{\rho}_{\mu \nu}^{\text {corr }}$, but fortunately it is sufficient to consider four elements only. Introducing local particle and hole operators $\hat{p}_{\mu}=|1\rangle_{\mu}\langle 2|$ and $\hat{h}_{\mu}=|0\rangle_{\mu}\langle 1|$, we find that their correlation functions $f_{\mu \nu}^{11}=\left\langle\hat{h}_{\mu}^{\dagger} \hat{h}_{\nu}\right\rangle$, $f_{\mu \nu}^{12}=\left\langle\hat{h}_{\mu}^{\dagger} \hat{p}_{\nu}\right\rangle, f_{\mu \nu}^{21}=\left\langle\hat{p}_{\mu}^{\dagger} \hat{h}_{\nu}\right\rangle$, and $f_{\mu \nu}^{22}=\left\langle\hat{p}_{\mu}^{\dagger} \hat{p}_{\nu}\right\rangle$, obey for $\nu \neq \mu$ a closed linear system of equations

$$
\begin{array}{r}
\left(i \partial_{t}+V_{\mu}-V_{\nu}-U\right) f_{\mu \nu}^{12}=-\frac{J \sqrt{2}}{Z} T_{\mu \nu}- \\
-\frac{J}{Z} \sum_{\kappa \neq \mu, \nu} T_{\mu \kappa}\left[3 f_{\kappa \nu}^{12}+\sqrt{2} f_{\kappa \nu}^{22}+\sqrt{2} f_{\kappa \nu}^{11}\right], \\
\left(i \partial_{t}+V_{\mu}-V_{\nu}\right) f_{\mu \nu}^{11}=\left(i \partial_{t}+V_{\mu}-V_{\nu}\right) f_{\mu \nu}^{22}= \\
-\frac{\sqrt{2} J}{Z} \sum_{\kappa \neq \mu, \nu} T_{\mu \kappa}\left(f_{\kappa \nu}^{21}-f_{\kappa \nu}^{12}\right),
\end{array}
$$

together with the symmetry $f_{\mu \nu}^{12}=\left(f_{\nu \mu}^{21}\right)^{*}$. Apart from this trivial relation, we find an effective particle hole symmetry $f_{\mu \nu}^{11}=f_{\mu \nu}^{22}$ (to first order in $1 / Z$ ).

The Analogy. Eqs. (9) provide a complete set of equations whose solution yields the number of particle-hole pairs created by the lattice tilt. However, instead of solving them directly, we make the following trick. It turns out that we may recover (i.e., factorize) Eqs. (9) to first order in $1 / Z$ if we assume the following effective linear equations of the operators $\hat{h}_{\mu}$ and $\hat{p}_{\mu}$

$$
\begin{aligned}
& {\left[i \partial_{t}-V_{\mu}-\frac{U}{2}\right] \hat{p}_{\mu}=-\frac{J}{Z} \sum_{\nu} T_{\mu \nu}\left[\frac{3}{2} \hat{p}_{\nu}+\sqrt{2} \hat{h}_{\nu}\right],} \\
& {\left[i \partial_{t}-V_{\mu}+\frac{U}{2}\right] \hat{h}_{\mu}=\frac{J}{Z} \sum_{\nu} T_{\mu \nu}\left[\frac{3}{2} \hat{h}_{\nu}+\sqrt{2} \hat{p}_{\nu}\right],(10)}
\end{aligned}
$$

and exploit the initial conditions $\left\langle\hat{h}_{\mu}^{\dagger} \hat{h}_{\nu}\right\rangle_{0}=\delta_{\mu \nu}$ and $\left\langle\hat{h}_{\mu}^{\dagger} \hat{p}_{\nu}\right\rangle_{0}=\left\langle\hat{p}_{\mu}^{\dagger} \hat{h}_{\nu}\right\rangle_{0}=\left\langle\hat{p}_{\mu}^{\dagger} \hat{p}_{\nu}\right\rangle_{0}=0$ in the Mott state. Note that these are effective equations of motion - they are not obtained by $i \partial_{t} \hat{h}_{\mu}=\left[\hat{h}_{\mu}, \hat{H}\right]$ etc. Nevertheless, the two-point functions in Eqs. (9) behave as if the operators $\hat{h}_{\mu}$ and $\hat{p}_{\mu}$ evolve according to (10). In this sense, solving Eqs. (10) yields the correct physics.

As the next step, we consider the (continuum) limit of large length scales and approximate the tunneling matrix

$$
\frac{1}{Z} \sum_{\nu} T_{\mu \nu} \hat{h}_{\nu}(t) \approx\left[1+\xi \nabla^{2}+\mathcal{O}\left(\nabla^{4}\right)\right] \hat{h}(t, \boldsymbol{r})
$$


by a long-wavelength expansion, where $\xi=\mathcal{O}(1 / Z)$ is the stiffness. Introducing effective scalar fields $\hat{\phi}_{ \pm}(t, \boldsymbol{r})=$ $\hat{h}(t, \boldsymbol{r}) \pm \hat{p}(t, \boldsymbol{r})$, we thus find the set of equations

$$
\left(i \partial_{t}-V\right) \hat{\phi}_{ \pm}=\frac{1}{2}[(3 \mp 2 \sqrt{2}) \mathcal{D}-U] \hat{\phi}_{\mp}
$$

where $\mathcal{D}=J\left[1+\xi \nabla^{2}+\mathcal{O}\left(\nabla^{4}\right)\right]$. As we shall see later, a very small tilt of the lattice will only induce a significant probability for pair creation if we are close to the critical point: $U=J(3+2 \sqrt{2})+\varepsilon$ with $0<\varepsilon \ll U$. In this case, the derivative part $\xi \nabla^{2}$ can be neglected in the term $(3-2 \sqrt{2}) \mathcal{D}$ and the coupled equations (12) simplify to

$$
\left(i \partial_{t}-V\right)^{2} \hat{\phi}_{+}=\left[m_{\mathrm{eff}}^{2} c_{\mathrm{eff}}^{4}-c_{\mathrm{eff}}^{2} \boldsymbol{\nabla}^{2}+\mathcal{O}\left(\boldsymbol{\nabla}^{4}\right)\right] \hat{\phi}_{+} .
$$

Up to short-distance corrections $\mathcal{O}\left(\nabla^{4}\right)$, we obtain the analogue of the Klein-Fock-Gordon equation (2) with an effective light velocity $c_{\text {eff }}^{2}=\xi\left(3 J U-J^{2}\right) / 2$ [12] and an effective mass $m_{\text {eff }}$ which is related to the energy gap

$$
\Delta \mathcal{E}=\sqrt{J^{2}-6 J U+U^{2}}=2 m_{\mathrm{eff}} c_{\mathrm{eff}}^{2}
$$

of the Mott insulator (in analogy to the energy gap $2 m_{e} c^{2}$ between the Dirac sea and the positive energy solutions). Even if we are not close to the critical point, the above equation (13) should remain a good approximation since the ratio $(3-2 \sqrt{2}) /(3+2 \sqrt{2}) \approx 0.03 \ll 1$ of the two numerical factors in (12) happens to be quite small.

Particle-hole pair creation. For potentials of the form $V_{\mu}(t)=q \boldsymbol{r}_{\mu} \cdot \boldsymbol{E}(t)$, it is convenient to apply a Fourier transform after shifting the potential $V_{\mu}(t)$ into the phase

$$
\hat{h}_{\mu}(t) e^{i \int_{0}^{t} d t^{\prime} V_{\mu}\left(t^{\prime}\right)}=\sum_{\boldsymbol{k}} \hat{h}_{\boldsymbol{k}}(t) e^{i \boldsymbol{k} \cdot \boldsymbol{r}_{\mu}},
$$

and analogously for $\hat{p}_{\mu}(t)$. Then Eqs. (10) become

$$
\begin{aligned}
i \partial_{t} \hat{h}_{\boldsymbol{k}} & =+\frac{1}{2}\left[3 J T_{\boldsymbol{k}}-U\right] \hat{h}_{\boldsymbol{k}}+\sqrt{2} J T_{\boldsymbol{k}} \hat{p}_{\boldsymbol{k}}, \\
i \partial_{t} \hat{p}_{\boldsymbol{k}} & =-\frac{1}{2}\left[3 J T_{\boldsymbol{k}}-U\right] \hat{p}_{\boldsymbol{k}}-\sqrt{2} J T_{\boldsymbol{k}} \hat{h}_{\boldsymbol{k}},
\end{aligned}
$$

where the $T_{k}$ are now time-dependent

$$
\frac{Z}{N} \sum_{\boldsymbol{k}} T_{\boldsymbol{k}} e^{i \boldsymbol{k} \cdot\left(\boldsymbol{r}_{\mu}-\boldsymbol{r}_{\nu}\right)}=T_{\mu \nu} e^{-i \int_{0}^{t} d t^{\prime}\left[V_{\mu}\left(t^{\prime}\right)-V_{\nu}\left(t^{\prime}\right)\right]} .
$$

Inserting $V_{\mu}(t)=q \boldsymbol{r}_{\mu} \cdot \boldsymbol{E}(t)=\partial_{t}\left[q \boldsymbol{r}_{\mu} \cdot \boldsymbol{A}(t)\right]$, we see that the time dependence of $T_{k}$ can be understood as replacing $\boldsymbol{k}$ by $\boldsymbol{k}+q \boldsymbol{A}(t)$. This is also known as Peierls substitution and is completely analogous to the gauge transformation in electrodynamics discussed between Eqs. (11) and (2). It also underlies the well-known Bloch oscillations.

The most general solution of (16) can be written as

$$
\begin{aligned}
& \hat{h}_{\boldsymbol{k}}(t)=f_{\boldsymbol{k}}^{+}(t) \hat{A}_{\boldsymbol{k}}+f_{\boldsymbol{k}}^{-}(t) \hat{B}_{\boldsymbol{k}}, \\
& \hat{p}_{\boldsymbol{k}}(t)=g_{\boldsymbol{k}}^{+}(t) \hat{A}_{\boldsymbol{k}}+g_{\boldsymbol{k}}^{-}(t) \hat{B}_{\boldsymbol{k}},
\end{aligned}
$$

with suitable functions $f_{\boldsymbol{k}}^{ \pm}(t)$ and $g_{\boldsymbol{k}}^{ \pm}(t)$ and operators $\hat{A}_{k}$ and $\hat{B}_{k}$. In the initial stationary regime where $J=V_{\mu}=0$, we have $\hat{h}_{\boldsymbol{k}}(t)=\hat{h}_{\boldsymbol{k}}^{\text {in }} \exp \{i U t / 2\}$ and $\hat{p}_{\boldsymbol{k}}(t)=\hat{p}_{\boldsymbol{k}}^{\text {in }} \exp \{-i U t / 2\}$. Thus, w.l.o.g. we may identify $\hat{h}_{\boldsymbol{k}}^{\text {in }}$ with $\hat{A}_{\boldsymbol{k}}$ and $\hat{p}_{\boldsymbol{k}}^{\text {in }}$ with $\hat{B}_{\boldsymbol{k}}$. This implies $f_{\boldsymbol{k}}^{+}(t) \propto$ $\exp \{i U t / 2\}$ and $g_{\boldsymbol{k}}^{-}(t) \propto \exp \{-i U t / 2\}$ initially with $g_{\text {in }}^{+}=f_{\text {in }}^{-}=0$. During the time evolution according to (16), however, $f_{\boldsymbol{k}}^{ \pm}(t)$ and $g_{\boldsymbol{k}}^{ \pm}(t)$ will mix and positive and negative frequencies will not stay separated as discussed after Eq. (3). In the final state (where again $\left.J=V_{\mu}=0\right)$, we have $\hat{h}_{\boldsymbol{k}}(t)=\hat{h}_{\boldsymbol{k}}^{\text {out }} \exp \{i U t / 2\}$ and $\hat{p}_{\boldsymbol{k}}(t)=\hat{p}_{\boldsymbol{k}}^{\text {out }} \exp \{-i U t / 2\}$ once more. Consequently, initially and finally the positive or negative frequency components of the field $\hat{\phi}_{\boldsymbol{k}}^{+}(t)=\hat{h}_{\boldsymbol{k}}(t)+\hat{p}_{\boldsymbol{k}}(t)$ in Eq. (13) yield the initial and final particle or hole operators, respectively. As a result, a mixing of positive and negative frequencies of the $\hat{\phi}_{+}$-field in Eq. (13) as given by the Bogoliubov coefficients $\alpha_{\boldsymbol{k}}$ and $\beta_{\boldsymbol{k}}$ directly corresponds to a mixing of particle and hole operators

$$
\hat{p}_{\boldsymbol{k}}^{\text {out }}=\alpha_{\boldsymbol{k}} \hat{p}_{\boldsymbol{k}}^{\text {in }}+\beta_{\boldsymbol{k}} \hat{h}_{\boldsymbol{k}}^{\text {in }} .
$$

In Fourier space, the initial conditions discussed after Eq. (10) read $\left\langle\hat{p}_{\boldsymbol{k}}^{\dagger} \hat{p}_{\boldsymbol{k}}\right\rangle_{\text {in }}=\left\langle\hat{p}_{\boldsymbol{k}}^{\dagger} \hat{h}_{\boldsymbol{k}}\right\rangle_{\text {in }}=\left\langle\hat{h}_{\boldsymbol{k}}^{\dagger} \hat{p}_{\boldsymbol{k}}\right\rangle_{\text {in }}=0$ and $\left\langle\hat{h}_{\boldsymbol{k}}^{\dagger} \hat{h}_{\boldsymbol{k}}\right\rangle_{\text {in }}=1$. Thus, inserting (19), we get

$$
\left\langle\hat{p}_{\boldsymbol{k}}^{\dagger} \hat{p}_{\boldsymbol{k}}\right\rangle_{\text {out }}=\left|\beta_{\boldsymbol{k}}\right|^{2} .
$$

This determines the on-site particle/hole probability via $\left\langle 2\left|\hat{\rho}_{\mu}\right| 2\right\rangle_{\text {out }}=\left\langle\hat{p}_{\mu}^{\dagger} \hat{p}_{\mu}\right\rangle_{\text {out }}=\sum_{\boldsymbol{k}}\left|\beta_{\boldsymbol{k}}\right|^{2} / N=\left\langle 0\left|\hat{\rho}_{\mu}\right| 0\right\rangle_{\text {out }}$ according to Eq. (7) and the aforementioned particlehole symmetry $f_{\mu \nu}^{11}=f_{\mu \nu}^{22}$. As expected from the exact analogy between Eqs. (2) and (13), we infer the same exponential scaling as in the Sauter-Schwinger effect (4)

$$
\left|\beta_{\boldsymbol{k}}\right|^{2} \propto \exp \left\{-\pi \frac{(\Delta \mathcal{E})^{2} / 4+c_{\mathrm{eff}}^{2} \boldsymbol{k}_{\perp}^{2}}{|\nabla V| c_{\mathrm{eff}}}\right\}
$$

for small $\boldsymbol{k}$ and small and slowly varying lattice tilts. Note that this expression is non-perturbative in $J$ and $U$, we only exploited the $1 / Z$-expansion.

Bloch oscillations. The exact analogy established above applies to small lattice tilts and large length scales. For large potential gradients, the long-wavelength expansion is not applicable anymore and the lattice structure becomes important. For simplicity, we assume a square lattice in the following, with the gradient pointing along a lattice axis, but the results can easily be generalized. For large tilts, one obtains resonance effects which can be understood by considering the Fourier transform of Eqs. (9) with the time-dependent $T_{\boldsymbol{k}}$ from Eq. (17)

$$
\begin{aligned}
\left(i \partial_{t}-U+3 J T_{k}\right) f_{k}^{12} & =-\sqrt{2} J T_{k}\left(f_{k}^{11}+f_{k}^{22}+1\right), \\
i \partial_{t} f_{\boldsymbol{k}}^{11}=i \partial_{t} f_{\boldsymbol{k}}^{22} & =\sqrt{2} J T_{\boldsymbol{k}}\left(f_{\boldsymbol{k}}^{12}-f_{\boldsymbol{k}}^{21}\right),
\end{aligned}
$$

where $f_{k}^{21}=\left(f_{k}^{12}\right)^{*}$. For a constant gradient $\nabla V$, the $T_{k}$ obey an oscillatory time-dependence due to the periodicity in $\boldsymbol{k} \rightarrow \boldsymbol{k}+t \boldsymbol{\nabla V}$, which is the basis of the well-known 
Bloch oscillations [5, 8]. For simplicity, we first consider the limit of small $J$ which facilitates a perturbative solution of Eqs. (22). To lowest oder in $J$, we obtain resonant growth if the potential difference $\Delta V$ between two neighbouring lattice sites equals the gap $\Delta \mathcal{E}=U+\mathcal{O}(J)$

$$
f_{\boldsymbol{k}}^{12}=\frac{i J t}{2 \sqrt{2}} e^{-i U t}+\mathcal{O}\left(J^{2}\right) \leadsto f_{\boldsymbol{k}}^{11}=\frac{J^{2} t^{2}}{8}+\mathcal{O}\left(J^{3}\right)
$$

This resonance has a width of $\sqrt{2} J$ and corresponds to tunneling to the nearest neighbouring site. In higher orders in $J$, we also obtain resonant tunneling to next-tonearest neighbours for $2 \Delta V=\Delta \mathcal{E}$ and so on. In contrast to the non-perturbative result (21), this process is more similar to electron-positron pair creation in the perturbative multi-photon regime, see, e.g., [2].

Beyond perturbation theory in $J$, we may employ Floquet analysis [9] to find the various resonance bands which yield an exponential growth of the solutions to Eqs. (22). The associated Floquet exponent for the first resonance at $\Delta V=\Delta \mathcal{E}$ reads $J /(\sqrt{2} \Delta V)$ and for the second resonance $2 \Delta V=\Delta \mathcal{E}$, it is $3 J^{2} /\left(4 \sqrt{2}[\Delta V]^{2}\right)$ etc.

Conclusions. In summary, we demonstrated that electron-positron pair production by a strong (and slowly varying) electric field and particle-hole pair creation in a slightly tilted Mott insulating Bose-Hubbard lattice are governed by the same equations. This quantitative analogy is sketched in the following table:

\begin{tabular}{|c|c|}
\hline Sauter-Schwinger effect & Bose-Hubbard model \\
\hline electrons \& positrons & particles \& holes \\
Dirac sea & Mott state \\
mass of electron/positron & energy gap $\Delta \mathcal{E}$ \\
electric field $\boldsymbol{E}$ & lattice tilt $V_{\mu}$ \\
speed of light $c$ & velocity $c_{\mathrm{eff}}$ \\
\hline
\end{tabular}

This analogy allows us to apply the vast machinery developed for the Sauter-Schwinger effect [1 -3$]$ to condensed matter theory. For example, small timedependent variations of the potential gradient may significantly enhance the tunneling probability [3] even if the rate of change is much smaller than the energy gap $\Delta \mathcal{E}$. These effects should be observable with atoms in optical lattices [4] where the experimental technique now even allows the in situ detection of single atoms [10]. Finally, it would be interesting to study whether this quantitative analogy can be extented to the onset of dielectric breakdown in the fermionic Hubbard model, see, e.g., [1].
This work was supported by the SFB/TR 12 of the German Research Foundation (DFG). Helpful discussions with K. Krutitsky are gratefully acknowledged.

* ralf.schuetzhold@uni-due.de

[1] F. Sauter, Zeits f. Physik 69, 742 (1931); J. Schwinger, Phys. Rev. 82, 664 (1951); L. V. Keldysh, Sov. Phys. JETP 02, 1307 (1965).

[2] E. Brezin and C. Itzykson, Phys. Rev. D 2, 1191 (1970); G. V. Dunne, Eur. Phys. J. D, 55, 327 (2009).

[3] R. Schützhold, H. Gies, and G. Dunne, Phys. Rev. Lett. 101, 130404 (2008).

[4] M. Greiner, O. Mandel, T. Esslinger, T. W. Hänsch et al., Nature 415, 39 (2002); J. Zakrzewski, Phys. Rev. A 71, 043601 (2005); I. Bloch, Nature Physics, 1, 23 (2005); C. Sias, A. Zenesini, H. Lignier, S. Wimberger et al., Phys. Rev. Lett. 98, 120403 (2007); M. Raizen, C. Salomon, and Q. Niu, Physics Today, 50, 30 (1997).

[5] O. Morsch, J. H. Müller, M. Cristiani, D. Ciampini et al., Phys. Rev. Lett. 87, 140402 (2001); Phys. Rev. A 65, 063612 (2002); M. B. Dahan, E. Peik, J. Reichel, Y. Castin et al., Phys. Rev. Lett. 76, 4508 (1996).

[6] P. Navez and R. Schützhold, Phys. Rev. A 82, 063603 (2010).

[7] R. Kubo, J. Phys. Soc. Japan 17, 1100 (1962); R. Balescu, Equilibrium and Nonequilibrium Statistical Mechanics (Wiley, New York, 1975).

[8] D. Witthaut, M. Werder, S. Mossmann, and H. J. Korsch, Phys. Rev. E 71, 036625 (2005); S. Sachdev, K. Sengupta, and S. M. Girvin, Phys. Rev. B 66, 075128 (2002); S. Wimberger, R. Mannella, O. Morsch, E. Arimondo et al., Phys. Rev. A 72, 063610 (2005); A. R. Kolovsky and H. J. Korsch, Phys. Rev. A 67, 063601 (2003); Int. J. Mod. Phys. B 18, 1235 (2004); A. R. Kolovsky, Phys. Rev. A 70, 015604 (2004); Phys. Rev. Lett. 90, 213002 (2003); A. R. Kolovsky and A. Buchleitner, Phys. Rev. E 68, 056213 (2003); A. R. Kolovsky, H. J. Korsch and E.-M. Graefe, Phys. Rev. A 80023617 (2009); D.-I. Choi and Q. Niu, Phys. Rev. Lett. 82, 2022 (1999).

[9] Z. X. Wang and D. R. Guo, Special Functions (World Scientific, 1989).

[10] P. Würtz, T. Langen, T. Gericke, A. Koglbauer et al., Phys. Rev. Lett. 103, 080404 (2009); W. S. Bakr, A. Peng, M. E. Tai, J. Simon et al., Science 329, 547 (2010); N. Gemelke, X. Zhang, C.-L. Hung, and C. Chin, Nature 460, 995 (2009); J. F. Sherson, C. Weitenberg, M. Endres, M. Cheneau et al., Nature 467, 68 (2010).

[11] M. Eckstein, T. Oka, and P. Werner, Phys. Rev. Lett. 105, 146404 (2010); T. Oka, R. Arita, and H. Aoki, Phys. Rev. Lett. 91, 066406 (2003); T. Oka and H. Aoki, Phys. Rev. B 81, 033103 (2010).

[12] The expression for $c_{\text {eff }}$ in [6] contained a typo. 